\title{
Recomendaciones para procedimientos quirúrgicos durante la pandemia de COVID-19 (SARS-CoV-2). Liderazgo hacia el retorno a lo electivo
}

\author{
COVID-19 (SARS-CoV-2) recommendations for surgical procedures. Leadership towards \\ elective surgery
}

\author{
Carlos A. Morales-Morales ${ }^{1}$, Mauricio González-Urquijo ${ }^{1}$, Arturo J. Barahona-Iglesias, \\ Juan M. Abdo-Francis ${ }^{2,3}$, Sergio R. Sobrino-Cossío ${ }^{2,4}$ y Francisco J. Bosques-Padilla ${ }^{5 *}$ \\ ${ }^{1}$ Escuela de Medicina y Ciencias de la Salud, Tecnológico de Monterrey, Monterrey, Nuevo León; ${ }^{2}$ Centro Avanzado en Endoscopía y Estudios \\ Funcionales, Gástrica, SA de CV, Ciudad de México; ${ }^{3}$ Servicio de Endoscopía, Hospital Ángeles Acoxpa, Ciudad de México; ${ }^{4}$ Servicio de \\ Endoscopía, Hospital Ángeles del Pedregal, Ciudad de México; ${ }^{5}$ Departamento de Gastroenterología, Facultad de Medicina y Hospital Universitario, \\ Universidad Autónoma de Nuevo León, Monterrey, Nuevo León. México
}

\begin{abstract}
Resumen
A finales de diciembre de 2019, la COVID-19, una nueva enfermedad emergente, rápidamente se propagó en Wuhan, China. La Organización Mundial de la Salud la declaró formalmente una pandemia y emergencia sanitaria el 11 de marzo de 2020. El objetivo de este artículo es precisar y enumerar los puntos clave en relación a las recomendaciones emitidas por los distintos colegios, y sociedades quirúrgicas globales, para beneficio de la comunidad médica y quirúrgica mexicana. De acuerdo con la evidencia cientifica, se realizan recomendaciones para las consultas médicas, los procedimientos quirúrgicos y endoscópicos, la infraestructura hospitalaria y los servicios de cirugía, además de una propuesta a la reapertura para procedimientos quirúrgicos en torno a la pandemia de COVID-19. El momento de tomar el liderazgo en salud en el que el sistema nacional de salud y las sociedades académicas, las universidades y la iniciativa privada sumen esfuerzos para combatir la pandemia ha llegado. Es conveniente formar grupos de colaboración de expertos en las distintas especialidades que, por medio de innovación en salud y educación, apego a la medicina basada en la evidencia, eficiencia de costos operacionales y herramientas como la telemedicina, permitan regresar a los procedimientos quirúrgicos cotidianos y la operación de los servicios de cirugía se reestablezca a la brevedad.
\end{abstract}

Palabras clave: COVID-19. Pandemia. SARS-CoV2.

\begin{abstract}
In late December 2019, COVID-19, a new emerging disease, quickly spread in Wuhan, China. The WHO formally declared it a pandemic and a health emergency on March $11^{\text {th }}, 2020$. The objective of this article is to specify and list key points in relation to the recommendations issued by the different colleges, and global surgical societies, for the benefit of the Mexican medical and surgical community. Based on scientific evidence, we make recommendations for medical consultations, surgical and endoscopic procedures, hospital infrastructure, and surgical services, in addition to a proposal to reopen surgical services and elective procedures related to the COVID-19 pandemic. The time to take leadership in healthcare where the national health
\end{abstract}

\section{Correspondencia:}

*Francisco J. Bosques-Padilla

Avenida Madero y Gonzalitos, s/n

Col. Mitras

Fecha de recepción: 23-06-2020

C.P. 64700 , Monterrey, N.L., México

E-mail: fbosques58@ hotmail.com

DOI: 10.24875/CIRU.20000692

0009-7411/@ 2020 Academia Mexicana de Cirugía. Publicado por Permanyer. Este es un artículo open access bajo la licencia CC BY-NC-ND (http://creativecommons.org/licenses/by-nc-nd/4.0/).
Cir Cir. 2020;88(6):794-798

Contents available at PubMed

www.cirugiaycirujanos.com 
system together with academic societies, universities and private initiative join forces to combat the pandemic has arrived. It is convenient to form collaboration groups of experts in the different specialties that through innovation in health and education, with evidence-based medicine, efficiency of operational costs and tools such as telemedicine, allow us to return to daily surgical procedures, reestablishing the surgery services as soon as possible.

Key words: COVID-19. Pandemic. SARS-CoV2.

\section{Introducción}

A finales de diciembre de 2019, la COVID-19, una nueva enfermedad emergente producida por el SARS-CoV-2, rápidamente se propagó en Wuhan, China. La Organización Mundial de la Salud (OMS) la declaró formalmente una pandemia y emergencia sanitaria el 11 de marzo de 20201,2. Debido a la propagación global, los sistemas de salud y sus trabajadores se han enfrentado a retos que evidencian la vulnerabilidad de estos. La cirugía es un pilar fundamental de la atención a la salud en la que recae la decisión de priorizar intervenciones quirúrgicas, establecer áreas de trabajo COVID-19 y no-COVID-19, formar equipos de trabajo y garantizar precauciones universales de protección ${ }^{3}$. México se ha unido para aplanar la curva de las infecciones y hospitalizaciones por esta enfermedad, y aunque la batalla está lejos de ganarse, no debemos olvidar que hay también un creciente sufrimiento de los pacientes vulnerables con enfermedades crónicas.

\section{Objetivo y planteamiento del problema}

Los informes nos dibujan un panorama nefasto en el sistema de salud estadounidense, agotado por los pacientes de COVID-19, que será incapaz de tratar el flujo normal de pacientes con enfermedades crónicas. Consideremos, por ejemplo, los procedimientos de reemplazo de la válvula aórtica para ciertos pacientes con insuficiencia cardíaca. A medida que los quirófanos y los laboratorios de cateterismo cardíaco se cierran, y tanto las visitas como las operaciones se cancelan, un gran número de pacientes ya no reciben ni están siendo evaluados para un reemplazo de la válvula aórtica. Sin embargo, la tasa de mortalidad de los pacientes que esperan el reemplazo por 6 meses es del $23 \%$, y sigue aumentando mientras la intervención se retrasa ${ }^{4}$.

Hasta ahora ha sido difícil cuantificar el exceso de muertes por la enfermedad subyacente, pero una publicación con datos del National Records of Scotland permite ver esto con más detalle. Ellos definen el exceso como la diferencia entre las muertes registradas en 2020 y el promedio de muertes en los 5 años anteriores. El estudio muestra un aumento significativo, de 38 muertes adicionales por cáncer (8,4 por millón de adultos), 103 muertes de causa circulatoria (22,8 por millón de adultos) y 100 por otras causas, y 83 muertes adicionales por demencia/Alzheimer (80 por millón en adultos mayores de 65 años) $)^{5}$. Posponer la cirugía de un paciente oncológico causaría que el promedio de años de vida ganados por paciente atendido, que habitualmente es de 18 en condiciones estándar, se reduciría de 9 a 15 con el retraso de 6 meses (una pérdida promedio de 2-3 años de vida por paciente) ${ }^{6}$.

Debido al entusiasmo de generar información valiosa para combatir la pandemia, la información científica y las recomendaciones para los procedimientos quirúrgicos durante la pandemia de COVID-19 han sido dinámicas y abundantes. El objetivo de este artículo es precisar y enumerar los puntos clave en tiempo real en relación con las recomendaciones emitidas por los distintos colegios y sociedades quirúrgicas globales, para beneficio de la comunidad médica y quirúrgica mexicana. Hay que tener en consideración que los tiempos epidemiológicos y el momento de intervención son distintos a los de Italia, España o los Estados Unidos de América.

\section{Consultas médicas}

Las consultas de primera vez y seguimiento se han tenido que reagendar, y en caso de ser urgente se puede optar por consulta a distancia por telemedicina o agendando la cita con previa intervención de la administración hospitalaria para preguntar por síntomas específicos de la enfermedad sugestivos de COVID-19, como fiebre, tos o cefalea ${ }^{7}$, sin perder de vista las condiciones médico-quirúrgicas que requieren atención inmediata.

La necesidad de reconversión hospitalaria para liberar camas y ventiladores, y por consiguiente la reubicación de personal médico y de enfermería, han disminuido la capacidad de mantener el personal quirúrgico completo, siendo uno de los múltiples motivos para decretar la suspensión de cirugías no urgentes y no oncológicas?. 


\section{Recomendación a los servicios de cirugía}

Al día de hoy, la Society of American Gastrointestinal and Endoscopic Surgeons y la European Association of Endoscopic Surgery recomiendan que todos los procedimientos quirúrgicos y endoscópicos electivos sean postergados, pero como mencionamos previamente, deben ser adaptados a la necesidad local. En preparación al aumento exponencial de casos de COVID-19, la atención quirúrgica debe limitarse a los padecimientos que pongan en riesgo la vida del paciente; esto incluye pacientes con enfermedades malignas de rápido progreso o con síntomas que requieran cirugía urgente. De igual manera, se deberá proteger al personal de salud no escencial enviándolos a casa o trabajando en telemedicina ${ }^{8}$. La European Society for Trauma and Emergency Surgery recomienda que a todos los pacientes previos a cirugía se les realice una prueba diagnóstica de COVID-19 por reacción en cadena de la polimerasa. En caso de retraso del resultado se deberá manejar a los pacientes como casos sospechosos de acuerdo con las guías establecidas por la OMS. Los pacientes con síntomas sugestivos de COVID-19 deberán ser sometidos a radiografía, ultrasonido o tomografía computada de tórax en busca de neumonitis intersticial bilateral sugestiva de COVID-199. Se deben discutir con el paciente los riesgos potenciales de infección y sus consecuencias ${ }^{8}$.

\section{Recomendaciones durante los procedimientos quirúrgicos $y$ endoscópicos}

Todos los miembros designados a los procedimientos quirúrgicos deben contar con el equipo completo de protección personal (EPP). Se debe minimizar el uso de instrumentos de energía por el riesgo de aerosolización, pues si bien no ha demostrado asociación con la transmisión del virus, se mantiene como una teoría de contagio razonable ${ }^{7}$. Deben usarse preferiblemente sistemas con filtros de partículas ultrapequeñas durante la cirugía de mínima invasión. ${ }^{8}$ En caso de no contar con estos sistemas, se puede optar por conectar el sistema del neumoperitoneo directo a la succión del quirófano ${ }^{10}$.

Debido a la presencia de RNA viral en las heces, los procedimientos de endoscopía gastrointestinal son considerados de alto riesgo y su personal de salud debe reunir las mismas directrices de protección personal que los neumólogos, los intervencionistas, los anestesiólogos y los terapistas. El servicio de endoscopía gastrointestinal debe limitarse solo a emergencias, como sangrados, vólvulos, ictericia obstructiva, colangitis o manejo de cuerpos extraños ${ }^{11}$. La vigilancia y el tamizaje de pacientes asintomáticos, las colonoscopías después de 1 año de retirar un pólipo y las endoscopías de vigilancia, como el caso de los pacientes con Helicobacter pylori, deben ser pospuestas ${ }^{12}$.

Las recomendaciones de la European Society of Gastrointestinal Endoscopy en caso de realizar endoscopías una vez seleccionados los pacientes candidatos incluyen la desinfección de los endoscopios flexibles con desinfectantes con actividad virucida. Se hace incapié en no reutilizar material bajo ninguna circunstancia ${ }^{13}$. Si se realizan endoscopías en pacientes con sospecha o diagnóstico confirmado de COVID-19, el material de EPP debe ser como mínimo cubrebocas N95, batas quirúrgicas, caretas, doble guante y, si es posible, cubrebotas. El procedimiento idealmente debe realizarse en cuartos con presión negativa, con la excepción de los pacientes de terapia intensiva, en quienes la recomendación es realizarlo a un lado de la cama ${ }^{12-14}$. La American Society for Gastrointestinal Endoscopy sugiere hacer una prueba serológica o de reacción en cadena de la polimerasa para COVID-19 al menos 48 horas antes del procedimiento endoscópico programado ${ }^{15}$; además, no recomienda el uso de anestésicos tópicos, como lidocaína, para evitar el riesgo de aerosolización, y propone, en casos de alto riesgo, la intubación orotraqueal para evitar la fuga de aerosoles ${ }^{16}$. Una vez terminada la intervención, se deben desinfectar las superficies debido a que el virus puede permanecer en ellas hasta 3 días. El equipo deberá ser descontaminado de acuerdo con las guías establecidas y el material residual se desechará acorde con los lineamientos de COVID-19 en su área médica. En recuperación, los pacientes deberán permanecer en cuartos separados para evitar el riesgo de transmisión. El seguimiento de los pacientes deberá realizarse a los 7 días por telemedicina para evaluar el probable contagio de COVID-19 ${ }^{17}$.

\section{Requerimientos de infraestructura}

Es indispensable contar con un equipo multidisciplinario a cargo de los protocolos COVID-19 en las unidades de salud, que incluya personal médico y de enfermería capacitado sobre los lineamientos de COVID-19, equipo administrativo y de logística, y asesores académicos que modifiquen en tiempo real las dinámicas recomendaciones globales, para poder garantizar los siguientes tres principios fundamentales: 
- Un sistema de reconversión hospitalaria activo, con áreas designadas COVID-19 y no-COVID-19 para limitar el flujo de personal y de pacientes potencialmente contaminados.

- Garantizar que el personal de salud cuente con el equipo necesario de protección personal y la infraestructura precisa para realizar procedimientos con la mínima posibilidad de contagio. Vigilar el uso y el retiro correcto del EPP, lavado de manos y todos los lineamientos que van más allá del objetivo de este artículo.

- La capacidad operativa para la obtención y el uso de pruebas diagnósticas en todos los paciente que se sometan a un procedimiento quirúrgico.

De no contar con lo anterior, nuestra recomendación es que el servicio sea suspendido y esperar los tiempos para la reapertura paulatina.

\section{Propuesta para la reapertura}

Los pacientes con una enfermedad crónica no son un grupo uniforme. Es imperativo centrarse primero en las personas con mayor riesgo, teniendo en cuenta factores tanto socioeconómicos como clínicos:

- Factores socioeconómicos: los determinantes sociales han sido destacados como un factor de riesgo clave de la COVID-19. Nuestro sistema de salud sufre de inequidades en la calidad de la atención, y esta pandemia ha evidenciado en forma importante estas terribles disparidades.

- Factores clínicos: los perfiles o la gravedad de la enfermedad de algunos pacientes conllevan un mayor riesgo de tener resultados adversos. Además, los pacientes que están bien manejados con terapias eficaces que mitigan estos riesgos se enfrentan a un riesgo menor que los que no lo están. Es difícil imaginar que al paciente diabético o hipertenso mal controlado no le irá peor con COVID-19 que al paciente que, a pesar de estar etiquetado con estas enfermedades, tiene valores normales de glucosa y de presión arterial. El manejo óptimo de las enfermedades crónicas mitigará los riesgos clínicos conocidos de estas condiciones y disminuirá el daño colateral que estamos viendo durante la pandemia. Idealmente, un manejo clínico óptimo se traducirá en mejores resultados y menos hospitalizaciones para aquellos que están infectados.

Considerando lo anterior, y con base en la evidencia científica, consideramos que para lograr el regreso de las actividades cotidianas de los servicios de cirugía es necesario que las sociedades y los colegios de especialidades, en común acuerdo con los Estados, logren definir qué procedimientos son urgentes y cuáles son no urgentes, como se logró en Massachusetts y Alaska en los Estados Unidos de América $^{18,19}$. Posterior a esto, hay que proponer un sistema de regreso paulatino de los procedimientos no urgentes acorde a la fase de la pandemia en su respectiva localidad. Esto deberá garantizar la correcta selección de casos con apego a la seguridad del paciente durante el preoperatorio y el posoperatorio. Un ejemplo propuesto para el manejo de pacientes quirúrgicos de alto riesgo, como son los pacientes bariátricos, es realizar un triaje del momento oportuno para un procedimiento quirúrgico tomando en cuenta factores clínicos que justifiquen una intervención expedita: «la priorización de cualquier procedimiento electivo debe buscar facilitar el acceso al procedimiento acorde a la necesidad clínica, maximizar equidad al acceso y minimizar el riesgo de retraso al procedimiento ${ }^{20}$.

De igual forma, es importante implementar formularios que faciliten el triaje de los pacientes que requieran procedimientos quirúrgicos, como es el caso del MeNTS (Medically Necessary Time Sensitive) propuesto por el American College of Surgeons, que objetivamente redirige el flujo de pacientes para mantener una eficiencia en el uso de ventiladores y terapias intensivas ${ }^{21}$, o el ESAS (Elective Surgery Acuity Scale) para el manejo de cirugías no urgentes ${ }^{22}$.

\section{Conclusiones}

Indudablemente, el virus ha cambiado la forma en que vivimos. Ha llegado el momento de tomar el liderazgo en salud, en el cual el sistema nacional de salud, con las sociedades académicas, las universidades y la iniciativa privada, sumen esfuerzos para combatir la pandemia.

Es urgente unificar y filtrar el flujo de la información científica nacional para emitir directrices en el ámbito quirúrgico. Es conveniente formar grupos de colaboración de expertos en las distintas especialidades que, por medio de innovación en salud y educación, apego a la medicina basada en la evidencia, eficiencia de costos operacionales y herramientas como la telemedicina, permitan regresar a los procedimientos quirúrgicos cotidianos y que la operación de los servicios de cirugía se reestablezca a la brevedad. La OMS ha hecho hincapié en que los sistemas de salud 
deben tener la capacidad de operar a lo largo de una doble vía, para que continúen prestando servicios de salud regulares, al tiempo que se responde agresivamente a la COVID-19. Es evidente que tanto los servicios de salud como la sociedad en su conjunto han sido puestos en acción para hacer frente a la COVID-19, y esto ha tenido un efecto en la prestación de otros servicios de salud y en las personas que acceden a dichos servicios. Debemos estar alerta y abiertos a nuevas formas de prestar atención médica. No podemos arriesgarnos a ver la tragedia de la COVID-19 reflejada en una segunda catástrofe de mala salud por otras causas.

Tal vez el mayor desafío, entonces, es invisible: ¿cómo ayudamos a las personas que tienen miedo de buscar atención médica? Esto se convierte en un reto para prácticamente todas las especialidades y áreas de la práctica médica moderna, algo que tendremos que resolver de manera innovadora e inteligente.

El mensaje final para todos nosotros es que la COVID-19 no va a desaparecer pronto. Los sistemas de salud de doble vía pueden ofrecer la flexibilidad y la resiliencia necesarias para gestionar las repetidas oleadas de infecciones por SARS-CoV-2 y la creciente demanda de otros servicios. Debemos atender la emergencia sanitaria creando las condiciones necesarias para el retorno de las actividades médicas y quirúrgicas habituales en el marco de una nueva normalidad.

\section{Financiamiento}

Este estudio no recibió ningún apoyo específico por parte de agencias de fondos, en el sector público, comercial o sin fines de lucro.

\section{Conflicto de intereses}

Los autores declaran no tener conflicto de intereses en la redacción de este manuscrito.

\section{Responsabilidades éticas}

Protección de personas y animales. Los autores declaran que para esta investigación no se han realizado experimentos en seres humanos ni en animales.

Confidencialidad de los datos. Los autores declaran que han seguido los protocolos de su centro de trabajo sobre la publicación de datos de pacientes.

Derecho a la privacidad y consentimiento informado. Los autores han obtenido el consentimiento informado de los pacientes y/o sujetos referidos en el artículo. Este documento obra en poder del autor de correspondencia.

\section{Bibliografía}

1. Sohrabi C, Alsafi Z, O'Neill N, Kahn M, Kerwan A, Al-Jabir, et al. World Health Organization declares global emergency: a review of the 2019 novel coronavirus (COVID-19). Int J Surg. 2020;76:71-76.

2. Yang W, Wang C, Shikora S, Kow L. Recommendations for metabolic and bariatric surgery during the COVID-19 pandemic from IFSO. Obes Surg. 2020;30:2071-3.

3. Flemming S, Hankir M, Ernestus RI, Seyfried F, Germer CT, Meybohm P, et al. Surgery in times of COVID-19 - recommendations for hospital and patient management. Langenbeck's Arch Surg. 2020;405:359-64.

4. Malaisrie SC, McDonald E, Kruse J, et al. Mortality while waiting for aortic valve replacement. Ann Thorac Surg. 2014;98:1564-71.

5. National Records of Scotland. Deaths involving coronavirus (COVID-19) in Scotland week 20 (11 to 17 May 2020). Consultado 30 mayo 2020. Disponible en https://www.nrscotland.gov.uk/covid19stats.

6. Sud A, Jones M, Broggio J, Loveday C, Torr B, Garrett A, et al. Collateral damage: the impact on outcomes from cancer surgery of the COVID-19 pandemic. Ann Oncol. 2020 May 19;S0923-7534(20)39825-2. doi: 10.1016/j.annonc.2020.05.009. Online ahead of print.

7. Mowbray NG, Ansell J, Horwood J, Cornish J, Rizkallah P, Parker A, et al. Safe management of surgical smoke in the age of COVID-19. Br J Surg. 2020 May 3;10.1002/bjs.11679. doi: 10.1002/bjs.11679. Online ahead of print.

8. Francis N, Dort J, Cho E, Feldman L, Keller D, Lim R, et al. SAGES and EAES recommendations for minimally invasive surgery during COVID-19 pandemic. Surg Endosc. 2020;34:2327-31.

9. Coimbra R, Edwards S, Kurihara H, Bass GA, Balogh ZJ, Tilsed J, et al. European Society of Trauma and Emergency Surgery (ESTES) recommendations for trauma and emergency surgery preparation during times of COVID-19 infection. Eur J Trauma Emerg Surg. 2020;46:505-10.

10. Hanbali N, Herrod P, Patterson J. A safe method to evacuate pneumoperitoneum during laparoscopic surgery in suspected COVID-19 patients. Ann R Coll Surg Engl. 2020;102:392-3.

11. Sinonquel $P$, Roelandt $P$, Demedts I, Van Gerven $L$, Vandenbriele $C$, Wilmer A, et al. COVID-19 and gastrointestinal endoscopy: what should be taken into account? Dig Endosc. 2020 Apr 26;10.1111/den.13706. doi: 10.1111/den.13706. Online ahead of print.

12. Furuta T, Irisawa A, Matsumoto T, Kawai T, Inaba T, Kanno A, et al. Clinical questions and answers on gastrointestinal endoscopy during the novel COVID-19 pandemic. Dig Endosc. 2020 May 29;10.1111/ den.13757. doi: 10.1111/den.13757. Online ahead of print.

13. Gralnek IM, Hassan C, Beilenhoff U, Antonelli G, Ebigbo A, Pellisè M, et al. ESGE and ESGENA Position Statement on gastrointestinal endoscopy and the COVID-19 pandemic. Endoscopy. 2020;52:483-90.

14. Ang TL. Gastrointestinal endoscopy during COVID-19 pandemic. J Gastroenterol Hepatol. 2020;35:701-2.

15. Hennesy B, Vicari J, Berstein B, Khaykis I, Littenber G, Robbins D, et al. Guidance for resuming $\mathrm{Gl}$ endoscopy and practice operations after the COVID-19 pandemic, Gastrointest Endosc. 2020. In press. doi: https:// doi.org/10.1016/j.gie.2020.05.006.

16. Thompson CC, Shen L, Lee LS. COVID-19 in endoscopy: Time to do more? Gastrointest Endosc. 2020;S0016-5107 (20)34126-2. doi:10.1016/j. gie.2020.03.3848.

17. Lui RN, Wong SH, Sánchez-Luna SA, et al. Overview of guidance for endoscopy during the coronavirus disease 2019 pandemic. J Gastroenterol Hepatol. 2020;35:749-59.

18. Menendez ME, Jawa A, Haas DA, Warner JJP, Comand Shoulder Society. Orthopedic Surgery Post COVID-19: An Opportunity for Innovation and Transformation, Journal of Shoulder and Elbow Surgery. 2020;29(6):1083-6.

19. Mouton C, Hirschmann MT, Ollivier M, Seil R, Menetrey J. COVID-19 - ESSKA guidelines and recommendations for resuming elective surgery. J Exp Orthop. 2020;7:28.

20. Rubino F, Cohen RV, Mingrone G, le Roux CW, Mechanick Jl, Arterburn DE, et al. Bariatric and metabolic surgery during and after the COVID-19 pandemic: DSS recommendations for management of surgical candidates and postoperative patients and prioritisation of access to surgery. Lancet Diabetes Endocrinol. 2020;8:1-9.

21. Prachand VN, Milner R, Angelos P, Posner MC, Fung JJ, Agrawal N, et al. Medically necessary, time-sensitive procedures: scoring system to ethically and efficiently manage resource scarcity and provider risk during the COVID-19 pandemic. J Am Coll Surg. 2020 Apr 9;S1072-7515(20)303173. doi: 10.1016/j.jamcollsurg.2020.04.011. Online ahead of print.

22. American College of Surgeons. COVID 19: elective case triage guidelines for surgical care. Disponible en: https://www.facs.org/covid-19/clinical-guidance/elective-case. 